TRANSACTIONS OF THE

AMERICAN MATHEMATICAL SOCIETY

Volume 350, Number 1, January 1998, Pages 165-183

S 0002-9947(98)02057-1

\title{
FACTORISATION IN NEST ALGEBRAS. II
}

\author{
M. ANOUSSIS AND E. G. KATSOULIS
}

\begin{abstract}
The main result of this paper is Theorem 5, which provides a necessary and sufficient condition on a positive operator $A$ for the existence of an operator $B$ in the nest algebra $A \lg N$ of a nest $N$ satisfying $A=B B^{*}$ (resp. $A=B^{*} B$ ). In Section 3 we give a new proof of a result of Power concerning outer factorisation of operators. We also show that a positive operator $A$ has the property that there exists for every nest $N$ an operator $B_{N}$ in $A l g N$ satisfying $A=B_{N} B_{N}^{*}$ (resp. $A=B_{N}^{*} B_{N}$ ) if and only if $A$ is a Fredholm operator. In Section 4 we show that for a given operator $A$ in $B(H)$ there exists an operator $B$ in $A \lg N$ satisfying $A A^{*}=B B^{*}$ if and only if the range $r(A)$ of $A$ is equal to the range of some operator in $A l g N$. We also determine the algebraic structure of the set of ranges of operators in $\operatorname{AlgN}$. Let $F_{r}(N)$ be the set of positive operators $A$ for which there exists an operator $B$ in $A l g N$ satisfying $A=B B^{*}$. In Section 5 we obtain information about this set. In particular we discuss the following question: Assume $A$ and $B$ are positive operators such that $A \leq B$ and $A$ belongs to $F_{r}(N)$. Which further conditions permit us to conclude that $B$ belongs to $F_{r}(N)$ ?
\end{abstract}

\section{INTRODUCTION AND PRELIMINARIES}

Let $H$ be a separable Hilbert space. A nest $N$ on $H$ is a totally ordered set of closed subspaces of $H$ containing $\{0\}$ and $H$ which is closed under intersection and closed span. The associated nest algebra $\operatorname{AlgN}$ is the set of bounded operators $A$ on $H$ leaving each member of $N$ invariant. The problem of factorisation of operators with respect to a nest $N$ consists in writing a positive operator $A$ in the form $B B^{*}$ (or $B^{*} B$ ) with $B$ in $A \lg N$. The factorisation of a positive invertible finite matrix $A$ as $B^{*} B$ with $B$ and its inverse in upper triangular form is known as the Cholesky decomposition. In [13] Gohberg and Krein obtain factorisations for operators which are compact perturbations of the identity with respect to arbitrary nests. Larson [15] studied factorisations of positive invertible operators $A$ in the form $B^{*} B$ with $B$ invertible in $A \lg N$. He showed that such a factorisation exists for every positive invertible operator if and only if the nest is countable. These results are concerned with factorisations of invertible or essentially invertible operators. Arveson [4] has introduced the concept of the outer operator in analogy with the outer functions in Hardy spaces. He has given a necessary and sufficient condition on a positive operator $A$ for the existence of a factorisation $A=B^{*} B$ with $B$ outer in $A l g N$, with respect to nests of a certain order type. Shields [23] obtained a factorisation for any positive trace class operator in the case of a nest of order type $\mathbb{N}$. In [20] Power, making a constructive approach, proved that every positive operator

Received by the editors January 24, 1996.

1991 Mathematics Subject Classification. Primary 47D25.

(C)1998 American Mathematical Society 
$A$ has a factorisation $A=B^{*} B$ with $B$ outer in $A \lg N$ if and only if the nest $N$ is well-ordered. Factorisation problems for other types of operator algebras which are related to nest algebras are also studied in [3],[17],[19]. Factorisation theory of operators is closely related to the theory of factorisation of positive operator-valued functions on the unit circle. We refer the reader to [18] for a survey of results and bibliography.

In this work we give a necessary and sufficient condition on a positive operator $A$ for the existence of an operator $B$ in the nest algebra $A l g N$ of a nest $N$ satisfying $A=B B^{*}$ (resp. $A=B^{*} B$ ). This result, which we prove in Section 2, holds for an arbitrary positive operator $A$ and for any nest $N$. If the nest $N$ is of order type $\mathbb{Z}$ the condition we give for the factorisation $A=B^{*} B$ is the same as the one given by Arveson in Theorem 3.3. of [4]. However in the general case the condition has a more elaborate form. The main idea in order to obtain the factorisation $A=B B^{*}$ is to consider the biggest projection $Q$ in $N$ which satisfies $Q=Q_{-}$and study the behaviour of the operator $A$ "near" $Q$. In Section 3 we use the technique developed in Section 2 to obtain a new proof of the above mentioned result of Power. We also show that a positive operator $A$ has the property that there exists for every nest $N$ an operator $B_{N}$ in $A l g N$ satisfying $A=B_{N} B_{N}^{*}$ (resp. $A=B_{N}^{*} B_{N}$ ) if and only if $A$ is a Fredholm operator. In Section 4 we show that for a given operator $A$ in $B(H)$ there exists an operator $B$ in $A l g N$ satisfying $A A^{*}=B B^{*}$ if and only if the range $r(A)$ of $A$ is equal to the range of some operator in $A l g N$ (Theorem 13). A consequence of this is that if $A$ and $C$ are positive operators in $B(H)$ with the same range, then there exists an operator $B$ in $\operatorname{Alg} N$ satisfying $A=B B^{*}$ if and only if there exists an operator $D$ in $A l g N$ satisfying $C=D D^{*}$. Theorem 13 motivates the study of the set of ranges of operators in $A l g N$, which we denote by $O R(N)$. In the rest of Section 4 we caracterise the nests $N$ for which the set $O R(N)$ is a meet semi-lattice (resp. a join semi-lattice). In Section 5 we consider a nest $N$ satisfying $I=I_{-}$and study the set of positive operators $A$ for which there exists an operator $B$ in $A l g N$ satisfying $A=B B^{*}$. This set is denoted by $F_{r}(N)$. We show that if $A$ is in $F_{r}(N)$ then $A^{\lambda}$ is in $F_{r}(N)$ for every positive number $\lambda$ with $0<\lambda \leq 1$, and show by an example that this is not true if $\lambda>1$. We also show that if $A$ and $C$ are in $F_{r}(N)$ then $A+C$ is in $F_{r}(N)$. A simple criterion is given which permits one to decide if an operator $A$ with closed range belongs to $F_{r}(N)$. We close Section 5 with Theorem 29, which provides a decomposition of a positive operator $A$ into a "factorable" and a "completely non-factorable" part with respect to a nest $N$ satisfying $I=I_{-}$. An analogous decomposition has been obtained in [21] and [1] for special cases.

As a general rule (with an exception in Theorem 10) we prove our results for the factorisation $B B^{*}$ and present the results concerning the factorisation $B^{*} B$ as corollaries. A reason for this choice is that it makes Theorem 13 appear more elegant.

Some of the results of this work (Theorem 5, Theorem 11 and Proposition 28) generalise previous results that we have obtained in [2] in the particular case of a continuous nest.

Throughout this work $H$ is a separable Hilbert space. The inner product on $H$ will be denoted by $\langle$,$\rangle . By a subspace of H$ we mean a subset of $H$ which is closed under addition of vectors and scalar multiplication. If $W$ is a subspace of $H, W^{\perp}$ is the subspace of $H$ consisting of the vectors orthogonal to each vector in $W$. If $V$ is a subset of $H,[V]$ is the linear span of $V$. If $\xi$ is a vector in $H,[\xi]$ is the linear 
span of $\xi$. If $\left\{V_{n}\right\}_{n=1}^{\infty}$ is a sequence of closed mutually orthogonal subspaces of $H$, we denote by $\sum_{n=1}^{\infty} \oplus V_{n}$ the closure of their linear span. We will denote by $B(H)$ the space of all bounded operators from $H$ into itself. Let $x, y$ be in $H$. The rank-one operator $x \otimes y$ is the operator on $H$ defined by: $z \rightarrow\langle z, x\rangle y$. If $A$ is in $B(H)$ we denote by $r(A)$ the range of $A$ and by coker $A$ the orthogonal complement of the kernel of $A$. If $P$ is an orthogonal projection in $B(H), P^{\perp}$ is the (orthogonal) projection $I-P$.

Let $N$ be a nest on $H$. The nest $N^{\perp}$ is defined to be $\left\{P^{\perp}: P \in N\right\}$. If $P$ is in $N$ we will denote by the same symbol the orthogonal projection on the subspace $P$. When the subspace $H$ (resp. $\{0\}$ ) is considered as an element of the nest $N$ it will be denoted by $I$ (resp. 0 ). If $E$ is a projection commuting with the elements of $N$, $E N$ is the nest in the Hilbert space $E H$ defined by $E N=\{E P: P \in N\}$. We will say that a vector $x$ in $H$ is $N$-proper if there exists a projection $P$ in $N, P \neq I$, such that $P x=x$. The set of $N$-proper vectors will be denoted by $\operatorname{Pr} N$. Given an element $P$ of $N$, we define $P_{-}$to be $\overline{\left[\bigcup_{L \in N, L<P} L\right]}$ and $P_{+}$to be $\bigcap_{L \in N, L>P} L$. We define $0_{-}$to be 0 and $I_{+}$to be $I$. The nest $N$ is continuous if $P=P_{-}$for every $P$ in $N$. The associated nest algebra $A l g N$ is the set of operators $A$ in $B(H)$ satisfying $P A P=A P$ for every $P$ in $N$. For a general discussion of nest algebras the reader is refered to [6].

We will say that a positive operator $A$ in $B(H)$ admits a right factorisation (resp. a left factorisation) with respect to $N$ if there exists an operator $B$ in $A l g N$ such that $A=B B^{*}\left(\right.$ resp. $\left.A=B^{*} B\right)$. We will denote by $F_{r}(N)\left(\operatorname{resp} . F_{l}(N)\right)$ the set of positive operators in $B(H)$ which admit a right factorisation (resp. a left factorisation) with respect to $N$.

\section{THE FACTORISATION THEOREM}

Throughout this section the letter $N$ will denote a nest on $H$, and $Q$ will be the element of $N$ defined by $Q=\bigcup_{P \in N, P=P_{-}} P$. Then it is easy to see that $Q=Q_{-}$and that for every $P$ in $N, P>Q$, we have $P \neq P_{-}$.

Lemma 1. Let $R$ be in $N$. Let $\left\{P_{n}\right\}_{n=0}^{\infty}$ be a sequence of elements of $N$ such that $P_{0}=0, P_{n+1}>P_{n}, P_{n} \neq R$ for each $n$, and $P_{n}$ converges strongly to $R$. Then there exists a sequence $\left\{M_{n}\right\}_{n=1}^{\infty}$ of closed mutually orthogonal infinite dimensional subspaces of $H$, such that $M_{n} \subset R \ominus P_{n}$ for every $n$.

Proof. Take for each $n$ a vector $e_{n}$ in $P_{n+1} \ominus P_{n}$ of norm 1. Take a sequence $\left\{A_{n}\right\}_{n=1}^{\infty}$ of mutually disjoint infinite subsets of $\mathbb{N}$. Put $M_{n}=\overline{\left[e_{m}: m \in A_{n}, m \geq n\right]}$.

Lemma 2. Let $A$ be an operator in $B(H)$. Let $R$ be in $N$. Let $\left\{P_{n}\right\}_{n=0}^{\infty}$ be a sequence of elements of $N$ such that $P_{0}=0, P_{n+1}>P_{n}, P_{n} \neq R$ for each $n$, and $P_{n}$ converges strongly to $R$. Then

$$
\sum_{n=1}^{\infty} \oplus\left(A^{-1}\left(P_{n}\right) \ominus A^{-1}\left(P_{n-1}\right)\right)=\overline{\bigcup_{P<R} A^{-1}(P) \cap \operatorname{coker} A} .
$$

Proof. For each $n$ the subspace $A^{-1}\left(P_{n}\right) \ominus A^{-1}\left(P_{n-1}\right)$ is contained in $A^{-1}\left(P_{n}\right) \cap$ coker $A$, which is in $\bigcup_{P<R} A^{-1}(P) \cap$ coker $A$. 
Let $P$ be in $N, P<R$. There exists $m$ such that $P<P_{m}$. We have $A^{-1}(P) \cap$ $\operatorname{coker} A \subset A^{-1}\left(P_{m}\right) \cap \operatorname{coker} A$. But

$$
A^{-1}\left(P_{m}\right) \cap \operatorname{coker} A=\sum_{n=1}^{m} \oplus\left(A^{-1}\left(P_{n}\right) \ominus A^{-1}\left(P_{n-1}\right)\right) .
$$

We conclude that $A^{-1}(P) \cap \operatorname{coker} A$ is contained in $\sum_{n=1}^{\infty} \oplus\left(A^{-1}\left(P_{n}\right) \ominus A^{-1}\left(P_{n-1}\right)\right)$.

Lemma 3. Let $A$ be an operator in $B(H)$. Then we have:

a) $\left(\sum_{P>Q} \oplus\left(A^{-1}(P) \ominus A^{-1}\left(P_{-}\right)\right)\right) \oplus A^{-1}(Q)=H$.

b) $\left(\sum_{P>Q} \oplus\left(A^{-1}(P) \ominus A^{-1}\left(P_{-}\right)\right)\right) \oplus\left(A^{-1}(Q) \cap \operatorname{coker} A\right)=\operatorname{coker} A$.

Proof. a) It is clear that the sum is orthogonal. Let $y$ be in $H$. Assume that $y$ is orthogonal to $\sum_{P>Q} \oplus\left(A^{-1}(P) \ominus A^{-1}\left(P_{-}\right)\right) \oplus A^{-1}(Q)$. Let $R=\inf \{P \in N: P A y=$ $A y\}$. We have $R A y=A y$. If $R>Q, y$ is orthogonal to $A^{-1}(R) \ominus A^{-1}\left(R_{-}\right)$; hence $y$ is in $A^{-1}\left(R_{-}\right)$. We conclude that $A y$ is in $R_{-}$, which is contrary to the definition of $R$. Therefore $R \leq Q$. But then $y$ is in $A^{-1}(Q)$ and is orthogonal to $A^{-1}(Q)$; hence $y=0$.

b) follows from a) and the fact that $\left(\sum_{P>Q} \oplus\left(A^{-1}(P) \ominus A^{-1}\left(P_{-}\right)\right)\right)$is contained in coker $A$.

Lemma 4. Let $L$ be in $N$ and $\left\{L_{n}\right\}_{n=0}^{\infty}$ be a sequence of elements of $N$ such that $L_{n+1}<L_{n}, L_{n} \neq L$ for each $n$ and $L_{n}$ converges strongly to $L$. Let $M$ be a closed subspace of $H$ contained in $L^{\perp}$. Assume that there exists $m$ in $\mathbb{N}$ such that $\operatorname{dim}\left(\left(\left(L_{n}\right)_{-}\right)^{\perp} \ominus\left(\left(\left(L_{n}\right)_{-}\right)^{\perp} \cap M\right)\right) \leq m$ for each $n$. Then $\operatorname{dim}\left(L^{\perp} \ominus M\right) \leq m$.

Proof. Assume $\operatorname{dim}\left(L^{\perp} \ominus M\right)>m$. Then there exist $m+1$ linearly independent vectors $x_{1}, x_{2}, \ldots, x_{m+1}$ in $L^{\perp} \ominus M$. For each $n$ the vectors $\left(\left(L_{n}\right)_{-}\right)^{\perp} x_{1},\left(\left(L_{n}\right)_{-}\right)^{\perp} x_{2}$, $\ldots,\left(\left(L_{n}\right)_{-}\right)^{\perp} x_{m+1}$ are orthogonal to $\left.\left(\left(L_{n}\right)_{-}\right)^{\perp} \cap M\right)$ and so their Grammian is 0 . The Grammians of the vectors $\left(\left(L_{n}\right)_{-}\right)^{\perp} x_{1},\left(\left(L_{n}\right)_{-}\right)^{\perp} x_{2}, \ldots,\left(\left(L_{n}\right)_{-}\right)^{\perp} x_{m+1}$ converge to the Grammian of the vectors $x_{1}, x_{2}, \ldots, x_{m+1}$. Hence the vectors $x_{1}, x_{2}, \ldots, x_{m+1}$ are linearly dependent.

Let $A$ be an operator in $B(H)$. We set

$$
n(A)=\operatorname{dim}\left(A^{-1}(Q) \ominus \overline{\bigcup_{P<Q} A^{-1}(P)}\right)
$$

if $Q \neq\{0\} ; n(A)=0$ if $Q=0$.

Let $P$ be in $N, P>Q$. We set $n_{P}(A)=+\infty$ if $\operatorname{dim}\left(P \ominus P_{-}\right)=+\infty$, and

$$
n_{P}(A)=\operatorname{dim}\left(P \ominus P_{-}\right)-\operatorname{dim}\left(A^{-1}(P) \ominus A^{-1}\left(P_{-}\right)\right)
$$

if $\operatorname{dim}\left(P \ominus P_{-}\right)<+\infty$. Note that $n_{P}(A) \geq 0$.

Theorem 5. Let $A$ be an operator in $B(H)$. The following are equivalent:

a) There exists an operator $B$ in AlgN such that $A A^{*}=B B^{*}$.

b) $\sum_{P>Q} n_{P}(A) \geq n(A)$. 
Proof. Assume b) holds. Consider for $P>Q$ a partial isometry $V_{P}$ with domain contained in $P \ominus P_{-}$and range $A^{-1}(P) \ominus A^{-1}\left(P_{-}\right)$. If $\operatorname{dim}\left(P \ominus P_{-}\right)=+\infty$, we choose $V_{P}$ in such a way that $\operatorname{dim}\left(\left(P \ominus P_{-}\right) \ominus \operatorname{dom} V_{P}\right)=+\infty$. Put $V_{1}=\sum_{P>Q} V_{p}$. Then $V_{1}$ is a partial isometry with range $\sum_{P>Q} \oplus\left(A^{-1}(P) \ominus A^{-1}\left(P_{-}\right)\right)$. We set $E_{P}=\left(P \ominus P_{-}\right) \ominus \operatorname{dom} V_{P}$.

Let $\left\{P_{n}\right\}_{n=0}^{\infty}$ be a sequence of elements of $N$ such that $P_{0}=0, P_{n+1}>P_{n}, P_{n} \neq$ $Q$ for each $n$, and $P_{n}$ converges strongly to $Q$. It follows from Lemma 1 that there exists a sequence $\left\{M_{n}\right\}_{n=1}^{\infty}$ of closed mutually orthogonal infinite dimensional subspaces of $H$, such that $M_{n} \subset Q \ominus P_{n}$ for every $n$. Consider for $n \geq 1$ a partial isometry $W_{n}$ with domain contained in $M_{n}$ and range $A^{-1}\left(P_{n}\right) \ominus A^{-1}\left(P_{n-1}\right)$. Put $V_{2}=\sum_{n=1}^{\infty} W_{n}$. Then $V_{2}$ is a partial isometry, and it follows from Lemma 2 that its range is $\bigcup_{P<Q} A^{-1}(P) \cap$ coker $A$.

Put $E=\sum_{P>Q} \oplus E_{P}$. Let $V_{3}$ be apartial isometry with domain contained in $E$ and range $A^{-1}(Q) \ominus \overline{\bigcup_{P<Q} A^{-1}(P)}$. Such an isometry exists, because

$$
\operatorname{dim} E=\sum_{P>Q} n_{P}(A) \geq \operatorname{dim}\left(A^{-1}(Q) \ominus \overline{\bigcup_{P<Q} A^{-1}(P)}\right)
$$

by hypothesis.

We set $V=V_{1}+V_{2}+V_{3}$. Then $V$ is a partial isometry with range

$\left(\sum_{P>Q} \oplus\left(A^{-1}(P) \ominus A^{-1}\left(P_{-}\right)\right)\right) \oplus\left(\overline{\bigcup_{P<Q} A^{-1}(P) \cap \operatorname{coker} A}\right) \oplus\left(A^{-1}(Q) \ominus \overline{\bigcup_{P<Q} A^{-1}(P)}\right)$.

Since

$$
A^{-1}(Q) \ominus \overline{\bigcup_{P<Q} A^{-1}(P)}=\left(\left(A^{-1}(Q) \cap \operatorname{coker} A\right) \ominus\left(\overline{\bigcup_{P<Q} A^{-1}(P) \cap \operatorname{coker} A}\right),\right.
$$

the range of $V$ is $\left.\underset{P>Q}{\left(\sum_{P}\right.} \oplus\left(A^{-1}(P) \ominus A^{-1}\left(P_{-}\right)\right)\right) \oplus\left(\left(A^{-1}(Q) \cap\right.\right.$ coker $\left.A\right)$, which by Lemma 3 is equal to coker $A$. We have $A=A V V^{*}$. We show that $A V$ is in $A l g N$. Let $R$ be in $N$ and $x$ be in $R$. We show that $A V x$ is in $R$. i) Assume $R<Q$. If $R \leq P_{1}, A V x=0$. If $R>P_{1}$, there exists $m \geq 1$ such that $P_{m}<R \leq P_{m+1}$. Then $A V x=A V_{2} x=A\left(\sum_{n=1}^{m} W_{n}\right) x$, which is contained in

$$
A\left(\sum_{n=1}^{m} \oplus\left(A^{-1}\left(P_{n}\right) \ominus A^{-1}\left(P_{n-1}\right)\right)\right) .
$$

But $A\left(\sum_{n=1}^{m} \oplus\left(A^{-1}\left(P_{n}\right) \ominus A^{-1}\left(P_{n-1}\right)\right)\right)$ is contained in $P_{m}$. Hence $A V x$ is in $R$. ii) Assume $R=Q$. We have $A V x=A\left(V_{2}+V_{3}\right) x$. But $r\left(V_{2}+V_{3}\right)$ is contained in $A^{-1}(Q)$. We conclude that $A V x$ is in $Q$. iii) Assume $R>Q$. Since $r\left(V_{2}\right.$ $\left.+V_{3}\right)$ is contained in $A^{-1}(Q)$ we see that $A\left(V_{2}+V_{3}\right) x$ is in $Q$. We have $A V_{1} x=$ $A\left(\sum_{Q<P \leq R} V_{p}\right) x$, which is contained in $A\left(\sum_{Q<P \leq R} \oplus\left(A^{-1}(P) \ominus A^{-1}\left(P_{-}\right)\right)\right)$. But $A\left(\sum_{Q<P \leq R} \oplus\left(A^{-1}(P) \ominus A^{-1}\left(P_{-}\right)\right)\right)$is contained in $R$. Hence $A V x$ is in $R$. 
Put $B=A V$. Then $B B^{*}=A V V^{*} A^{*}=A A^{*}$ and $B$ is in $A l g N$.

Assume a) holds. It follows from polar decomposition that there exists a partial isometry $U$ with domain coker $A$ and range $\operatorname{coker} B$ such that $A=B U$. Put $D=$ $\left(\bigcup_{P<Q} A^{-1}(P) \cap \operatorname{coker} A\right)$ and $M=\left(\left(A^{-1}(Q) \cap \operatorname{coker} A\right) \ominus\left(\overline{\bigcup_{P<Q}} A^{-1}(P) \cap \operatorname{coker} A\right)\right.$. We show that $U M$ is contained in $Q^{\perp}$. Take $m$ in $M$ and $P$ in $N, P<Q$. Since $r(A)=r(B)$ we have $B P U m=A x_{P}$ for some $x_{P}$ in coker $A$. Since $B P U m$ is in $P, x_{P}$ is in $A^{-1}(P) \cap \operatorname{coker} A$. We have $B P U m=B U x_{P}$, and so $P U m-U x_{P}$ is in $K e r B$. We have $P U m=P U m-U x_{P}+U x_{P}$, which belongs to $\operatorname{Ker} B \oplus U D$. We conclude that $Q U m=\lim _{P \in N, P \neq Q, P \rightarrow Q} P U m$ is in $\operatorname{Ker} B \oplus U D$. Since $U M$ is orthogonal to $\operatorname{Ker} B \oplus U D$, we see that $Q U m$ is orthogonal to $U m$. Therefore $U m$ is in $Q^{\perp}$.

We set $K_{P}=A^{-1}(P) \ominus A^{-1}\left(P_{-}\right)$for $P$ in $N, P>Q$. We show that $U K_{P}$ is contained in $\left(P_{-}\right)^{\perp}$. Let $x$ be in $K_{P}$. Since $r(A)=r(B)$ we have $B\left(P_{-}\right) U x=A y$ for some $y$ in $A^{-1}\left(P_{-}\right) \cap \operatorname{coker} A$. But then $B\left(P_{-}\right) U x=B U y$, and so $\left(P_{-}\right) U x-U y$ is in $\operatorname{Ker} B$. We have

$$
\left(P_{-}\right) U x=\left(P_{-}\right) U x-U y+U y,
$$

which is in $\operatorname{Ker} B \oplus U\left(A^{-1}\left(P_{-}\right) \cap \operatorname{coker} A\right)$. Since $U x$ is orthogonal to $\operatorname{Ker} B \oplus$ $U\left(A^{-1}\left(P_{-}\right) \cap \operatorname{coker} A\right)$ we see that $U x$ is orthogonal to $\left(P_{-}\right) U x$. Hence $\left(P_{-}\right) U x=0$.

Put $K=\sum_{P>Q} \oplus K_{P}$. Since $U M$ is contained in $Q^{\perp}$ and is orthogonal to $U K$, in order to prove b) it is enough to prove that $\operatorname{dim}\left(Q^{\perp} \ominus U K\right) \leq \sum_{P>Q} n_{P}(A)$. Put $\Omega=\{P \in N, P \geq Q\}, \Pi=\left\{P \in \Omega: \operatorname{dim}\left(\left(P_{-}\right)^{\perp} \ominus\left(\sum_{R \geq P} \oplus U K_{R}\right)\right) \leq \sum_{R \geq P} n_{R}(A)\right\}$, $\Sigma=\{P \in \Omega, P \notin \Pi\}$. It suffices to show that $\Sigma$ is empty.

Assume that $\Sigma \neq \emptyset$. Note that if $P$ is in $\Omega$ and is different from $Q$ then $P \neq P_{-}$. It follows that every non-empty subset of $\Omega$ has a maximum. Let $S$ be the maximum of $\Sigma$.

i) Suppose first that $S=L_{-}$for some $L$ in $\Pi$. Let $\pi$ be the canonical projection from $\left(S_{-}\right)^{\perp}$ onto $\left(S_{-}\right)^{\perp} /\left(\sum_{P \geq L} \oplus U K_{P}\right)$. Since $k e r \pi$ is contained in $\left(\sum_{P \geq S} \oplus U K_{P}\right)$, we see that

$$
\operatorname{dim}\left(\left(S_{-}\right)^{\perp} \ominus\left(\sum_{P \geq S} \oplus U K_{P}\right)\right)=\operatorname{dim} \pi\left(\left(S_{-}\right)^{\perp}\right)-\operatorname{dim} \pi\left(\sum_{P \geq S} \oplus U K_{P}\right) .
$$

We have

$$
\operatorname{dim} \pi\left(\left(S_{-}\right)^{\perp}\right)=\operatorname{dim} \pi\left(S \ominus S_{-}\right)+\operatorname{dim} \pi\left(\left(L_{-}\right)^{\perp}\right)
$$

and

$$
\operatorname{dim} \pi\left(\sum_{P \geq S} \oplus U K_{P}\right)=\operatorname{dim} U K_{S}
$$

So

$$
\begin{aligned}
\operatorname{dim}\left(\left(S_{-}\right)^{\perp} \ominus\left(\sum_{P \geq S} \oplus U K_{P}\right)\right) & =\operatorname{dim} \pi\left(S \ominus S_{-}\right)+\operatorname{dim} \pi\left(\left(L_{-}\right)^{\perp}\right)-\operatorname{dim} U K_{S} \\
& =\operatorname{dim}\left(S \ominus S_{-}\right)+\operatorname{dim} \pi\left(\left(L_{-}\right)^{\perp}\right)-\operatorname{dim} U K_{S} .
\end{aligned}
$$

But

$$
\operatorname{dim} \pi\left(\left(L_{-}\right)^{\perp}\right)=\operatorname{dim}\left(\left(L_{-}\right)^{\perp} \ominus\left(\sum_{P \geq L} \oplus U K_{P}\right)\right)
$$


and

$$
\operatorname{dim} \pi\left(\left(L_{-}\right)^{\perp}\right) \leq \sum_{P \geq L} n_{P}(A)
$$

since $L$ is in $\Pi$. We conclude that

$$
\operatorname{dim}\left(\left(S_{-}\right)^{\perp} \ominus\left(\sum_{P \geq S} \oplus U K_{P}\right)\right) \leq \sum_{P \geq L} n_{P}(A)+\operatorname{dim}\left(S \ominus S_{-}\right)-\operatorname{dim} U K_{S}=\sum_{P \geq S} n_{R}(A) .
$$

Hence $S$ is in $\Pi$, which is contrary to our assumption.

ii) Suppose that $S \neq L_{-}$for every $L$ in $\Pi$. Then there exists a sequence $\left\{L_{n}\right\}_{n=0}^{\infty}$ of elements of $\Pi$ such that $L_{n+1}<L_{n}, L_{n} \neq S$ for each $n$ and $L_{n}$ converges strongly to $S$. There exist finitely many $P>Q$ such that $n_{P}(A) \neq 0$. Put $m=\sum_{P>S} n_{P}(A)$. Then for each $n$ we have

$$
\begin{array}{r}
\operatorname{dim}\left(\left(\left(L_{n}\right)_{-}\right)^{\perp} \ominus\left(\left(\left(L_{n}\right)_{-}\right)^{\perp} \cap\left(\sum_{P>S} \oplus U K_{P}\right)\right)\right) \\
\leq \operatorname{dim}\left(\left(\left(L_{n}\right)_{-}\right)^{\perp} \ominus\left(\sum_{P \geq P_{n}} \oplus U K_{P}\right)\right) \leq m .
\end{array}
$$

It follows then from Lemma 4 that $\operatorname{dim}\left(S^{\perp} \ominus\left(\sum_{P>S} \oplus U K_{P}\right)\right) \leq m$. Let $\pi$ be the canonical projection from $\left(S_{-}\right)^{\perp}$ onto $\left(S_{-}\right)^{\perp} /\left(\sum_{P>S} \oplus U K_{P}\right)$. Proceeding as in (i), we see that

$$
\operatorname{dim}\left(\left(S_{-}\right)^{\perp} \ominus\left(\sum_{P \geq S} \oplus U K_{P}\right)\right) \leq \sum_{P \geq S} n_{P}(A) .
$$

Hence $S$ is in $\Pi$, which is contrary to our assumption.

We conclude that $\Sigma$ is empty.

If the nest $N$ has the property $I=I_{-}$, condition b) of Theorem 5 says that $A^{-1}(\operatorname{Pr} N)$ is dense in $H$. In this particular case this condition is essentially the same as the density condition given in Theorem 3.1 in [1] in a different but related context.

Corollary 6. Let $A$ be a positive operator in $B(H)$. Then $A$ admits a right factorisation with respect to $N$ if and only if $\sum_{P>Q} n_{P}\left(A^{\frac{1}{2}}\right) \geq n\left(A^{\frac{1}{2}}\right)$.

Let $R=\overline{\bigcap_{P \in N, P=P_{+}} P}$. Then it is easy to see that $R=R_{+}$and that for every $P$ in $N, P<R$, we have $P \neq P_{+}$. Let $A$ be an operator in $B(H)$. We set

$$
m(A)=\operatorname{dim}\left(\bigcap_{P>R} \overline{r(A P)} \ominus \overline{r(A R)}\right)
$$

if $R \neq H ; m(A)=0$ if $R=I$.

Let $P$ be in $N, P<R$. We set: $m_{P}(A)=+\infty$, if $\operatorname{dim}\left(P_{+} \ominus P\right)=+\infty$, and

$$
m_{P}(A)=\operatorname{dim}\left(P_{+} \ominus P\right)-\operatorname{dim}\left(\overline{r\left(A P_{+}\right)} \ominus \overline{r(A P)}\right)
$$

otherwise. Note that $m_{P}(A) \geq 0$.

Corollary 7. Let $A$ be an operator in $B(H)$. The following are equivalent:

a) There exists an operator $B$ in $A \lg N$ such that $A^{*} A=B^{*} B$.

b) $\sum_{P<R} m_{P}(A) \geq m(A)$. 
One should note that if the nest $N$ is of order type $\mathbb{Z}$ condition b) of Corollary 7 says that $\bigcap_{P>0} \overline{r(A P)}=\{0\}$. In this particular case this condition is the same as the one given by Arveson in Theorem 3.3. of [4]. A condition of the same type has been given by Lowdenslager in Theorem 1 of [16] in the context of factorisation of operator functions.

Corollary 8. Let $A$ be a positive operator in $B(H)$. Then $A$ admits a left factorisation with respect to $N$ if and only if $\sum_{P<R} m_{P}\left(A^{\frac{1}{2}}\right) \geq m\left(A^{\frac{1}{2}}\right)$.

\section{OUter FACTORISATION AND UNIVERSALly FACTORABLE OPERATORS}

Let $N$ be a nest on $H$. An operator $A$ in $A l g N$ is called outer if its range projection commutes with $N$ and $r(B P)$ is dense in $r(B) \cap P$ for every $P$ in $N$. Outer operators were introduced by Arveson in [4] in analogy with outer functions in Hardy spaces. Theorem 3.3 in [4] gives a necessary and sufficient condition on a positive operator $X$ for the existence of an outer operator $A$ in $A \lg N$ satisfying $X=A^{*} A$ under the assumption that the nest $N$ is of a certain order type. In [20] Power proves that for every positive operator $X$ in $B(H)$ there exists an outer operator $A$ in $A \lg N$ satisfying $X=A^{*} A$ if and only if the nest $N$ is well ordered. In what follows we give a proof of the result of Power based on the ideas of Section 2. Note that a nest $N$ is well ordered if and only if $P \neq P_{+}$for every $P$ in $N, P \neq I$.

Lemma 9. Let $N$ be a well-ordered nest on $H$ and $A$ be an operator in $B(H)$. Let $P_{0}$ be in $N$. Then

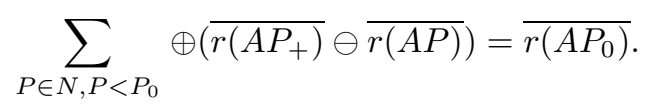

Proof. It is clear that $\left.\sum_{P \in N, P<P_{0}} \oplus \overline{\left(r\left(A P_{+}\right)\right.} \ominus \overline{r(A P)}\right)$ is contained in $\overline{r\left(A P_{0}\right)}$. Let $x$ be in $\overline{r\left(A P_{0}\right)}, x \neq 0$, and assume that $x$ is orthogonal to

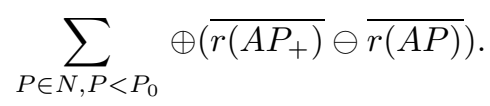

Put $S=\sup \{L \in N: x$ is orthogonal to $\overline{r(A L)}\}$. Then since $x \neq 0$ we get $S<$ $P_{0}$. Since $x$ is orthogonal to $\left(\overline{r\left(A S_{+}\right)} \ominus \overline{r(A S)}\right)$ we conclude that $x$ is orthogonal to $\overline{r\left(A S_{+}\right)}$, which is contrary to the definition of $S$. Therefore

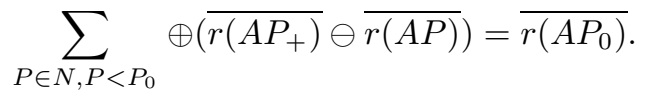

Theorem 10. a) Let $N$ be a well-ordered nest on $H$. Let $X$ be a positive operator in $B(H)$. Then there exists an outer operator $B$ in $A \lg N$ such that $X=B^{*} B$. Moreover, $B$ belongs to the von Neumann algebra generated by $X$ and the nest $N$.

b) Let $N$ be a nest on $H$. Assume that for every positive operator $X$ in $B(H)$ there exists an outer operator $B$ in $A l g N$ such that $X=B^{*} B$. Then $N$ is wellordered.

Proof. a) Put $A=X^{\frac{1}{2}}$. Let $P$ be in $N$. We denote by $M_{P}$ the orthogonal projection on $\overline{r\left(A P_{+}\right)} \ominus \overline{r(A P)}$. We set $A_{P}=M_{P} A\left(P_{+}-P\right)$. We show that $r\left(A_{P}\right)$ contains 
$r\left(A P_{+}\right) \cap r(A P)^{\perp}$. Let $y$ be in $r\left(A P_{+}\right) \cap r(A P)^{\perp}$. Then there exists $x$ in $P_{+}$such that $y=A x$. We have $y=A\left(P_{+}-P\right) x+A P x$, and therefore

$$
M_{P} y=M_{P} A\left(P_{+}-P\right) x+M_{P} A P x .
$$

Now since $A P x$ is contained in $r(A P)$ we have $M_{P} A P x=0$. Hence $y=M_{P} y=$ $M_{P} A\left(P_{+}-P\right) x$ and $y$ is in $r\left(A_{P}\right)$. Let $V_{P}\left|A_{P}\right|$ be the polar decomposition of $A_{P}$. Then $V_{P}$ is a partial isometry with domain contained in $P_{+} \ominus P$ and range $\overline{r\left(A_{P}\right)}$ which is equal to $\overline{r\left(A P_{+}\right)} \ominus \overline{r(A P)}$. Put $V=\sum_{P \in N} V_{P}$. We have

$$
\left.V V^{*}=\sum_{P \in N} \oplus \overline{\left(r\left(A P_{+}\right)\right.} \ominus \overline{r(A P)}\right),
$$

which is equal to $\overline{r(A)}$ by Lemma 9. Therefore $A=V V^{*} A$. We set $B=V^{*} A$. Then $X=B^{*} B$. Since $M_{P}$ lies in the von Neumann algebra generated by $X$ and the nest $N$, the same holds for the operators $V_{P}$ and hence also for $V$. We conclude that $B$ belongs to the von Neumann algebra generated by $X$ and the nest $N$. To finish the proof we have to show that $B$ is outer and lies in $A l g N$. The range of $\left(V_{P}\right)^{*} A$ is contained in $P_{+} \ominus P$, and hence the range projection of $\left(V_{P}\right)^{*} A$ commutes with $N$ for every $P$. It follows that the range projection of $B$ commutes with $N$. Let $P_{0}$ be in $N$. We will show now that $r\left(B P_{0}\right)$ is dense in $r(B) \cap P_{0}$. Let $y$ be in $r(B) \cap P_{0}$. Then $y=B x$ for some $x$ in $H$. We have $y=V^{*} A x$. Then $V y=A x$ and $A x$ is in $V P_{0}$, which is equal to

$$
\left.\sum_{P \in N, P<P_{0}} \oplus \overline{r\left(A P_{+}\right)} \ominus \overline{r(A P)}\right) .
$$

By Lemma 9, $\sum_{P \in N, P<P_{0}} \oplus\left(\overline{r\left(A P_{+}\right)} \ominus \overline{r(A P)}\right)$ is equal to $\overline{r\left(A P_{0}\right)}$. It follows that $V^{*} A x$ is in $V^{*} \overline{r\left(A P_{0}\right)}$. But $V^{*} \overline{r\left(A P_{0}\right)}$ is contained in $\overline{r\left(V^{*} A P_{0}\right)}=\overline{r\left(B P_{0}\right)}$. Therefore $y$ is in $\overline{r\left(B P_{0}\right)}$ and $r(B) \cap P_{0}$ is contained in $\overline{r\left(B P_{0}\right)}$. We conclude that $B$ is an outer operator in $\operatorname{Alg} N$.

b) Assume that $N$ is not well-ordered. Then there exists $P_{0}$ in $N$ such that $P_{0}=\left(P_{0}\right)_{+}$. Let $\xi$ be a unit vector in $H$ such that $\left(P_{0}\right) \xi=0$ and $P \xi \neq 0$ for every $P$ in $N, P>P_{0}$. Put $X=\xi \otimes \xi$. Assume there exists an outer operator $B$ in $A l g N$ such that at $X=B^{*} B$. The operator $B$ must be a rank one operator. So there exist vectors $x, y$ in $H$ such that $B=x \otimes y$. We have $\xi \otimes \xi=\langle y, y\rangle x \otimes x$, and hence $x$ is a multiple of $\xi$. It follows from the characterisation of the rank-one operators in $\operatorname{Alg} N$ given in [22] that $y$ belongs to $P_{0}$. But now we have $r\left(B P_{0}\right)=\{0\}$ and $r(B) \cap P_{0}=[y]$. We conclude that $B$ cannot be outer.

Now we are going to characterise the positive operators $A$ in $B(H)$ which admit a right factorisation (resp. a left factorisation) with respect to any nest $N$. We use some results from Fredholm theory which may be found in [5]. As in Section 2, if $N$ is a nest we denote by $Q$ the element of $N$ defined by $Q=\underset{P \in N, P=P_{-}}{\bigcup} P$.

Theorem 11. Let $A$ be an operator in $B(H)$.

a) There exists for every nest $N$ an operator $B_{N}$ in AlgN satisfying $A A^{*}=B_{N}$ $B_{N}^{*}$ if and only if $A$ is a right Fredholm operator.

b) There exists for every nest $N$ an operator $B_{N}$ in $\operatorname{Alg} N$ satisfying $A^{*} A=$ $B_{N}^{*} B_{N}$ if and only if $A$ is a left Fredholm operator. 
Proof. a) Assume that $A$ is a right Fredholm operator. Then $r(A)$ is closed and of co-finite dimension in $H$. Then $r(A) \cap Q$ is of co-finite dimension in $Q$. It follows from [2, Prop. 4] that $\bigcup_{P<Q} P \cap r(A)$ is dense in $r(A) \cap Q$. Since the restriction of $A$ onto coker $A$ is an isomorphism from coker $A$ onto $r(A)$, we see that the set $A^{-1}\left(\bigcup_{P<Q} P \cap r(A)\right) \cap \operatorname{coker} A$ is dense in $A^{-1}(Q \cap r(A)) \cap \operatorname{coker} A$. We have

$$
A^{-1}\left(\bigcup_{P<Q} P \cap r(A)\right) \cap \operatorname{coker} A=A^{-1}\left(\bigcup_{P<Q} P\right) \cap \operatorname{coker} A
$$

and

$$
A^{-1}(Q \cap r(A)) \cap \operatorname{coker} A=A^{-1}(Q) \cap \operatorname{coker} A .
$$

Since

$$
A^{-1}(Q) \ominus \overline{A^{-1}\left(\bigcup_{P<Q} P\right)}=\left(A^{-1}(Q) \cap \operatorname{coker} A\right) \ominus \overline{\left(A^{-1}\left(\bigcup_{P<Q} P\right) \cap \operatorname{coker} A\right)},
$$

we conclude that $n(A)=0$. It then follows from Theorem 5 that for every nest $N$ there exists an operator $B_{N}$ in $A l g N$ satisfying $A A^{*}=B_{N} B_{N}^{*}$.

Assume that for every nest $N$ there exists an operator $B_{N}$ in $A \lg N$ satisfying $A A^{*}=B_{N} B_{N}^{*}$. It follows from [2, Th. 15] that $A$ is a right Fredholm operator.

b) Assume that $A$ is a left Fredholm operator. Then $A^{*}$ is a right Fredholm operator. Let $N$ be a nest. It follows from a) that there exists an operator $C_{N^{\perp}}$ in $A \lg N^{\perp}$ satisfying $A^{*} A=C_{N^{\perp}} C_{N^{\perp}}^{*}$. Put $B_{N}=C_{N^{\perp}}^{*}$. Then $B_{N}$ is in $A \lg N$ and satisfies $A^{*} A=B_{N}^{*} B_{N}$.

Assume that for every nest $N$ there exists an operator $B_{N}$ in $A l g N$ satisfying $A^{*} A=B_{N}^{*} B_{N}$. It follows then from [2, Th. 15] that $A$ is a left Fredholm operator.

Corollary 12. Let $A$ be a positive operator in $B(H)$. Then $A$ admits a right factorisation (a left factorisation) with respect to every nest $N$ if and only if $A$ is a Fredholm operator.

Proof. It follows from Theorem 11 that $A$ admits a right factorisation with respect to every nest $N$ if and only if $A^{\frac{1}{2}}$ is a right Fredholm operator. Since $A^{\frac{1}{2}}$ is selfadjoint, $A^{\frac{1}{2}}$ is a right Fredholm operator if and only if it is a Fredholm operator. But $A^{\frac{1}{2}}$ is a Fredholm operator if and only if $A$ is a Fredholm operator.

The other assertion is proved in the same way.

\section{FACTORISATION AND RANGES OF OPERATORS}

Let $N$ be a nest on $H$. We set $O R(N)=\{W: W=r(X)$ for some $X$ in $A l g N\}$. Let $A$ be an operator in $B(H)$. Assume that there exists an operator $B$ in $A l g N$ such that $A A^{*}=B B^{*}$. Then $r(A)=r(B)$ by [10], and so $r(A)$ is in $O R(N)$. In Theorem 13 below we show that this condition is also sufficient for the existence of an operator $B$ in $A l g N$ satisfying $A A^{*}=B B^{*}$. As in Section 2, we denote by $Q$ the element of $N$ defined by $Q=\bigcup_{P \in N, P=P_{-}} P$.

Theorem 13. Let $N$ be a nest on $H$. Let $A$ be an operator in $B(H)$. The following are equivalent:

a) There exists an operator $B$ in AlgN such that $A A^{*}=B B^{*}$.

b) $r(A)$ is in $O R(N)$. 
Proof. It is clear that a) implies b).

b) implies a): Let $C$ be an operator in $\operatorname{Alg} N$ such that $r(A)=r(C)$. The operator $C$ clearly satisfies condition b) of Theorem 5 . We prove that so does $A$. For this it suffices to show that $n(A)=n(C)$ and $n_{P}(A)=n_{P}(C)$ for every $P>Q$ such that $\operatorname{dim}\left(P \ominus P_{-}\right)<+\infty$. It follows from [10] that there exists operators $X$ and $Y$ in $B(H)$ such that i) coker $X=\operatorname{coker} A, r(X)=\operatorname{coker} C$ and $A=C X$, ii) coker $Y=\operatorname{coker} C, r(Y)=\operatorname{coker} A$ and $C=A Y$. Then it is easy to see that $X Y x=x$ for every $x$ in coker $C$ and $Y X x=x$ for every $x$ in coker $A$.

We prove first that $n_{P}(A)=n_{P}(C)$ for every $P>Q$ such that $\operatorname{dim}\left(P \ominus P_{-}\right)<$ $+\infty$. Let $P$ be an element of $N$ such that $P>Q$ and $\operatorname{dim}\left(P \ominus P_{-}\right)<+\infty$, and $\pi$ be the canonical projection from $C^{-1}(P)$ onto $C^{-1}(P) / C^{-1}\left(P_{-}\right)$. Let $x$ be in $A^{-1}(P) \ominus A^{-1}\left(P_{-}\right)$. Then it is easy to see that $X x$ is in $C^{-1}(P)$. We are going to show that the linear map from $A^{-1}(P) \ominus A^{-1}\left(P_{-}\right)$to $C^{-1}(P) / C^{-1}\left(P_{-}\right)$defined by $x \rightarrow \pi(X x)$ is injective. In fact, if $\pi(X x)=0$, then $X x$ belongs to $C^{-1}\left(P_{-}\right)$ and $A x=C X x$ belongs to $P_{-}$. This implies that $x$ is in $A^{-1}\left(P_{-}\right)$, and hence it is 0 . It follows that $\operatorname{dim}\left(A^{-1}(P) \ominus A^{-1}\left(P_{-}\right)\right) \leq \operatorname{dim}\left(C^{-1}(P) \ominus C^{-1}\left(P_{-}\right)\right)$and hence $n_{P}(C) \leq n_{P}(A)$. A similar argument proves that $n_{P}(A) \leq n_{P}(C)$. We conclude that $n_{P}(C)=n_{P}(A)$ for every $P$ in $N$ such that $P>Q$ and $\operatorname{dim}\left(P \ominus P_{-}\right)<+\infty$.

We show now that $n(A)=n(C)$. Let $\pi$ be the canonical projection from $C^{-1}(Q)$ onto $C^{-1}(Q) \overline{C^{-1}(\operatorname{Pr} Q N)}$. Let $x$ be in $A^{-1}(Q) \ominus \overline{A^{-1}(\operatorname{Pr} Q N)}$. Then it is easy to see that $X x$ is in $C^{-1}(Q)$. We are going to show that the linear map from $A^{-1}(Q) \ominus \overline{A^{-1}(\operatorname{PrQN})}$ to $C^{-1}(Q) / \overline{C^{-1}(\operatorname{PrQN})}$ defined by $x \rightarrow \pi(X x)$ is injective. Assume $\pi(X x)=0$. Then $X x$ is in $\overline{C^{-1}(\operatorname{Pr} Q N)}$, and consequently there exists a sequence $w_{n}$ in $C^{-1}(\operatorname{Pr} Q N)$ converging to $X x$. Therefore the sequence $Y w_{n}$ converges to $Y X x$, which is equal to $x$. Since $w_{n}$ is in $C^{-1}(\operatorname{Pr} Q N), C w_{n}$ is in $\operatorname{Pr}(Q N)$. Since $A Y w_{n}=C w_{n}$, we conclude that $Y w_{n}$ is in $A^{-1}(\operatorname{Pr} Q N)$. Therefore $x$, being the limit of $Y w_{n}$, is in $\overline{A^{-1}(\operatorname{Pr} Q N)}$. Hence $x=0$. It follows that

$$
\operatorname{dim}\left(A^{-1}(Q) \ominus \overline{A^{-1}(\operatorname{Pr} Q N)}\right) \leq \operatorname{dim}\left(C^{-1}(Q) \ominus \overline{C^{-1}(\operatorname{Pr} Q N)}\right) .
$$

A similar argument shows that

$$
\operatorname{dim}\left(C^{-1}(Q) \ominus \overline{C^{-1}(\operatorname{Pr} Q N)}\right) \leq \operatorname{dim}\left(A^{-1}(Q) \ominus \overline{A^{-1}(\operatorname{Pr} Q N)}\right) .
$$

We conclude that $n(A)=n(C)$.

It follows now from Theorem 5 that there exists an operator $B$ in $\operatorname{Alg} N$ such that $A A^{*}=B B^{*}$.

Corollary 14. Let $N$ be a nest on $H$. Let $A$ and $C$ be operators in $B(H)$. We assume that $r(A)=r(C)$. Then there exists an operator $B$ in $\operatorname{Alg} N$ such that $A A^{*}=B B^{*}$ if and only if there exists an operator $D$ in $A \lg N$ such that $C C^{*}=$ $D D^{*}$.

Proof. This follows from Theorem 13.

Corollary 15. Let $N$ be a nest on $H$. Let $A$ and $C$ be positive operators in $B(H)$. We assume that $r(A)=r(C)$. Then:

a) The operator $A$ is in $F_{r}(N)$ if and only if the operator $C$ is in $F_{r}(N)$.

b) The operator $A$ is in $F_{l}(N)$ if and only if the operator $C$ is in $F_{l}(N)$.

Proof. a) Theorem 1 in [10] implies that there exist positive numbers $\lambda$ and $\mu$ such that $A^{2} \leq \lambda C^{2} \leq \mu A^{2}$. It follows then from [14, Prop. 4.2.8.] that $A \leq \lambda^{\frac{1}{2}} C \leq \mu^{\frac{1}{2}} A$. 
Hence, using again Theorem 1 of [10], we obtain $r\left(A^{\frac{1}{2}}\right)=r\left(C^{\frac{1}{2}}\right)$. Now the assertion follows from Corollary 14.

b) follows from a) and the fact that $F_{l}(N)=F_{r}\left(N^{\perp}\right)$.

In the rest of this section we study the set $O R(N)$. The importance of this set emerges from Theorem 13.

Let $\mathcal{S}$ be a set of subspaces of $H$ containing $\{0\}$ and $H$. We say that $\mathcal{S}$ is a join semi-lattice if whenever $V$ and $W$ are in $\mathcal{S}, V+W$ is in $\mathcal{S}$. We say that $\mathcal{S}$ is a meet semi-lattice if whenever $V$ and $W$ are in $\mathcal{S}, V \cap W$ is in $\mathcal{S}$. If $\mathcal{S}$ is a join semi-lattice and a meet semi-lattice we say that it is a lattice. When $N$ is the trivial nest consisting of the subspaces $\{0\}$ and $H$, the set $O R(N)$ is the set of ranges of operators in $B(H)$. In this case it was shown by Dixmier that $O R(N)$ is a lattice [8]. A proof of this result may also be found in [11] or [12]. In what follows we characterise the nests $N$ for which $O R(N)$ is a join or a meet semi-lattice.

Proposition 16. Let $N$ be a nest and $W$ a linear subspace of $H$. The following are equivalent:

a) The subspace $W$ is in $O R(N)$.

b) There exists an operator $A$ in $B(H)$ with $r(A)=W$ which satisfies condition b) of Theorem 5 .

c) Every operator $A$ in $B(H)$ with $r(A)=W$ satisfies condition b) of Theorem 5 .

Proof. It follows from Theorem 11 and Theorem 5 that a) implies c).

It is clear that c) implies b).

b) implies a) by Theorem 5 and Theorem 13 .

Corollary 17. Let $N$ be a nest. We assume that one of the following holds:

a) $Q=0$.

b) There exist $P$ in $N, P>Q$, such that $\operatorname{dim}\left(P \ominus P_{-}\right)=+\infty$.

Then $O R(N)$ is the set of ranges of operators in $B(H)$. In particular, $O R(N)$ is a lattice.

Proof. Let $A$ be an operator in $B(H)$. Then $A$ satisfies condition b) of Theorem 5, and it follows from Proposition 16 that $r(A)$ is in $O R(N)$.

Corollary 18. Let $N$ be a nest. Assume that $Q \neq 0$ and that for every $P$ in $N, P>Q$, we have $\operatorname{dim}\left(P \ominus P_{-}\right)<+\infty$. Let $\xi$ be a vector in $Q$ which is not $Q N$-proper. There is no operator in AlgN with range $Q^{\perp}+[\xi]$.

Proof. Put $B=Q^{\perp}+\xi \otimes \xi$. It is clear that $r(B)=Q^{\perp}+[\xi]$. It follows from Proposition 16 that $B$ must satisfy condition b) of Theorem 5 . But an easy calculation shows that $\sum_{P>Q} n_{P}(B)=0$ and $n(B)=1$. The conclusion follows.

Proposition 19. Let $N$ be a nest such that $I=I_{-}$. Then there exist $A, C$ in AlgN such that $r(A) \cap r(C)$ is not the range of any operator in AlgN .

Proof. A necessary and sufficient condition for a rank one operator $\eta \otimes \xi$ to lie in $A \lg N$ is that there exists $P$ in $N$ such that $\xi$ is in $P$ and $\eta$ is in $\left(P_{-}\right)^{\perp}$ [22]. Therefore, if $\xi$ is a vector in $H$ which is not $N$-proper, there is no operator in $A l g N$ with range $[\xi]$. So it suffices to construct operators $A$ and $C$ in $A l g N$ such that $r(A) \cap r(C)=[\xi]$ and $\xi$ is not $N$-proper.

Let $\left\{P_{n}\right\}_{n=0}^{\infty}$ be a sequence of elements of $N$ such that $P_{0}=0, P_{n+1}>P_{n}, P_{n} \neq$ $I, \operatorname{dim}\left(P_{n+1} \ominus P_{n}\right) \geq 2$ for each $n$, and $P_{n}$ converges strongly to $I$. We consider 
unit vectors $e_{n}, f_{n}$ in $P_{n+1} \ominus P_{n}$ with $e_{n}$ orthogonal to $f_{n}$. We define $w_{0}=f_{0}$, $w_{1}=2^{-1} f_{1}-f_{0}, w_{n}=2^{-n} f_{n}-\left(\sum_{i=0}^{n-1} 2^{-i} f_{i}\right)$ for each $n$. We consider the operators $A=\sum_{n=1}^{\infty} e_{n} \otimes e_{n-1}$ and $B=\sum_{n=1}^{\infty} 2^{-\frac{n}{2}} e_{n} \otimes w_{n-1}$. It is clear that $A$ and $B$ are in $A \lg N$. We set $C=A+B$, and we will prove that $A$ and $C$ are as required. Let $x$ be in $r(A) \cap r(C)$. Then there exist $y$ and $z$ in $H$ such that $x=A y=C z=A z+B z$. We have $B z=A(y-z)$, and since $r(A)$ and $r(B)$ are orthogonal we obtain that $B z=0$ and $z$ is in $\operatorname{ker} B$. It follows that $x=A y=A z$, and so $x$ is in $A(k e r B)$. Conversely, assume that $w$ is in $\operatorname{ker} B$. We have $A w=(A+B) w=C w$, and so $A($ ker $B)$ is contained in $r(A) \cap r(C)$. Thus $r(A) \cap r(C)=A(k e r B)$. We are going to show that the subspace $A(\operatorname{ker} B)$ is spanned by a vector which is not $N$-proper. Let $z$ be in $\operatorname{ker} B$. Then $z=\sum_{n=1}^{\infty} z_{n} e_{n}+z_{0} e_{0}+r$, where $z_{n}$ are complex numbers for $n=0,1,2, \ldots$ and $r$ is in $\left[\left\{e_{n}: n=0,1,2, \ldots\right\}\right]^{\perp}$. Since $B z=0$ we obtain $\sum_{n=1}^{\infty} 2^{-\frac{n}{2}} z_{n} w_{n-1}=0$. Now if $\sum_{n=0}^{\infty} \lambda_{n} w_{n}=0$, then taking scalar products with $f_{0}$, $f_{1}, \ldots$ successively, we find that $\lambda_{0}=\sum_{k=1}^{\infty} \lambda_{k}, \lambda_{1}=\sum_{k=2}^{\infty} \lambda_{k}, \ldots, \lambda_{n}=\sum_{k=n+1}^{\infty} \lambda_{k}$ for each $n$, and hence $\lambda_{0}-\lambda_{1}=\lambda_{1}, \lambda_{1}-\lambda_{2}=\lambda_{2}, \ldots \lambda_{n}-\lambda_{n+1}=\lambda_{n+1}$ for each $n$. Therefore $\lambda_{n}=2^{-n} \lambda_{0}$. It follows that $z_{n+1}=2^{-\frac{n}{2}} z_{1}$ for $n=1,2, \ldots$. We conclude that

$$
z=\sum_{n=1}^{\infty} z_{n} e_{n}+z_{0} e_{0}+r=z_{1}\left(\sum_{n=1}^{\infty} 2^{-\frac{n-1}{2}} e_{n}\right)+z_{0} e_{0}+r
$$

Since $z_{0} e_{0}+r$ is in $\operatorname{ker} A, A z=z_{1}\left(\sum_{n=1}^{\infty} 2^{-\frac{n-1}{2}} e_{n-1}\right)$. So the subspace $A(k e r B)$ is spanned by the vector $\sum_{n=1}^{\infty} 2^{-\frac{n-1}{2}} e_{n-1}$, which is not $N$-proper. We conclude that $A(\operatorname{ker} B)$ cannot be the range of any operator in $A l g N$.

Theorem 20. Let $N$ be a nest. Then $O R(N)$ is a meet semi-lattice if and only if one of the following cases occurs:

a) $Q=0$.

b) There exist $P$ in $N, P>Q$, such that $\operatorname{dim}\left(P \ominus P_{-}\right)=+\infty$.

Proof. Suppose that $Q \neq 0$ and for every $P$ in $N, P>Q$, we have $\operatorname{dim}\left(P \ominus P_{-}\right)$ $<+\infty$. We will show that $O R(N)$ is not a meet semi-lattice. It follows from Proposition 19 that there exist operators $A$ and $C$ in $A \lg Q N$ such that $r(A) \cap r(C)=$ $[\xi]$ and $\xi$ is not $Q N$-proper. We define $A_{1}=Q^{\perp}+A, C_{1}=Q^{\perp}+C$. Then $A_{1}$ and $C_{1}$ are in $A \lg N$ and $r\left(A_{1}\right) \cap r\left(C_{1}\right)=Q^{\perp}+[\xi]$. By Corollary 18, $Q^{\perp}+[\xi]$ is not in $O R(N)$.

Assume now that a) or b) holds. It follows from Corollary 17 that $O R(N)$ is the set of ranges of operators in $B(H)$, which is a lattice.

Lemma 21. Let $N$ be a nest such that $I=I_{-}$. Then there exist partial isometries $U_{1}$ and $U_{2}$ in $A l g N$ with orthogonal domains and such that $r\left(U_{1}\right)=r\left(U_{2}\right)=H$.

Proof. Let $\left\{P_{n}\right\}_{n=0}^{\infty}$ be a sequence of elements of $N$ such that: $P_{0}=0, P_{n+1}>$ $P_{n}, P_{n} \neq I$ for each $n$, and $P_{n}$ converges strongly to $I$. It follows from Lemma 1 that there exists a sequence $\left\{M_{n}\right\}_{n=1}^{\infty}$ of closed mutually orthogonal infinite dimensional 
subspaces of $H$ such that $M_{n} \subset\left(P_{n}\right)^{\perp}$ for every $n$. Let $V_{0}$ be a partial isometry with domain contained in $M_{1}$ and range $P_{1}$. For each $n \geq 1$, consider a partial isometry $V_{n}$ with domain contained in $M_{2 n+1}$ and range $P_{2 n+1} \ominus P_{2 n-1}$. Put $U_{1}=$ $\sum_{n=0}^{\infty} V_{n}$. For each $n \geq 2$, consider a partial isometry $W_{n}$ with domain contained in $M_{2 n}$ and range $P_{2 n} \ominus P_{2 n-2}$. Put $U_{2}=\sum_{n=1}^{\infty} W_{n}$.

Theorem 22. Let $N$ be a nest. Then $O R(N)$ is a join semi-lattice if and only if one of the following three cases occurs:

a) $Q=0$.

b) $Q=I$.

c) There exist $P$ in $N, P>Q$, such that $\operatorname{dim}\left(P \ominus P_{-}\right)=+\infty$.

Proof. Assume first that a) or c) holds. It follows from Corollary 17 that $O R(N)$ is a lattice. Assume that b) holds. Let $W_{1}, W_{2}$ be in $O R(N)$. Then $W_{1}=r\left(A_{1}\right)$, $W_{2}=r\left(A_{2}\right)$ for some operators $A_{1}, A_{2}$ in $A l g N$. It follows from Lemma 21 that there exist partial isometries $U_{1}$ and $U_{2}$ in $A l g N$ with orthogonal domains and such that $r\left(U_{1}\right)=r\left(U_{2}\right)=H$. We set $B=A_{1} U_{1}+A_{2} U_{2}$. Then $B$ is in $A l g N$ and $r(B)=W_{1}+W_{2}$.

Assume now that $Q \neq 0, Q \neq I$, and for every $P$ in $N, P>Q$, we have $\operatorname{dim}\left(P \ominus P_{-}\right)<+\infty$. We will show that $O R(N)$ is not a join semi-lattice. Put $A=Q^{\perp}$ and $B=e \otimes \xi$, where $e$ is in $Q^{\perp}$ and $\xi$ is a vector in $Q$ which is not $Q N$ proper. Then $A$ and $B$ are in $A \lg N$ and $r(A)+r(B)=Q^{\perp}+[\xi]$. By Corollary 18 there is no operator in $\operatorname{AlgN}$ with range $Q^{\perp}+[\xi]$.

\section{Nests With $I=I_{-}$}

Let $N$ be a nest on $H$. In this section we obtain information about the set $F_{r}(N)$ Let $A$ and $C$ be positive operators in $B(H)$. Consider the following condition:

(i) There exists a positive number $\lambda$ such that $A \leq \lambda C$.

Put $A_{1}=A^{\frac{1}{2}}, C_{1}=C^{\frac{1}{2}}$. By [10, Th.1] condition (i) is equivalent to the following condition:

(ii) There exists an operator $X$ in $B(H)$ such that $A_{1}=C_{1} X$, coker $X=\operatorname{coker} A_{1}$ and $r(X)$ is contained in coker $C_{1}$.

We are interested in the following question:

Question: Assume (i) holds. Assume that $A$ is in $F_{r}(N)$. Is it true that $C$ is in $F_{r}(N)$ ?

The above question in the generality stated has a negative answer. However if we assume moreover that the nest $N$ satisfies $I=I_{-}$and that the range of the operator $X$ is dense in $\operatorname{coker} C_{1}$, the answer is positive. This is shown in Theorem 23, below.

A similar question has been considered by Lowdenslager in [16] and by Douglas in [9] in the context of factorisation of operator functions. The results obtained there are used to prove Devinatz's Theorem [7].

The condition : "the range of the operator $X$ is dense in coker $C_{1}$ " is implicit in the work of Douglas [9]. Theorem 23 and Corollaries 24 and 25 below are motivated by that paper. We remark that Theorem 23 improves Corollary 14 in the case of a nest with the property $I=I_{-}$. 
Theorem 23. Let $A$ and $C$ be operators in $B(H)$. We assume that there exists an operator $X$ in $B(H)$ such that $A=C X$, coker $X=$ coker $A$ and $r(X)$ is contained in coker $C$. Then the following are equivalent:

a) $r(X)$ is dense in coker $C$.

b) i) $\overline{r(A)}=\overline{r(C)}$.

ii) Let $N$ be a nest on $H$ such that $I=I_{-}$. Assume that there exists an operator $B$ in $A \lg N$ such that $A A^{*}=B B^{*}$. Then there exists an operator $D$ in $A l g N$ such that $C C^{*}=D D^{*}$.

Proof. a) implies b).

i) We have $A^{*}=X^{*} C^{*}$, and since $r(X)$ is dense in $\operatorname{coker} C$ we obtain that $\operatorname{Ker} A^{*}=\operatorname{Ker} C^{*}$. It follows that $\overline{r(A)}=\overline{r(C)}$.

ii) The subspace $A^{-1}(\operatorname{Pr} N)$ is dense in $H$ by Theorem 5 . It follows that $X\left(A^{-1}(\operatorname{Pr} N)\right)$ is dense in $r(X)$ and hence in coker $C$. But $C^{-1}(\operatorname{Pr} N)$ contains $X\left(A^{-1}(\operatorname{Pr} N)\right)+\operatorname{ker} C$, which is dense in coker $C+\operatorname{ker} C=H$. Again by Theorem 5 we conclude that there exists an operator $D$ in $A \lg N$ such that $C C^{*}=D D^{*}$.

b) implies a).

Assume first that coker $A$ is of finite dimension. Then $\operatorname{dimr}(A)=\operatorname{dimr}(X)$ and by condition i) $\operatorname{dim} r(A)=\operatorname{dim} r(C)$. So $\operatorname{dim} r(X)=\operatorname{dim} r(C)$, and since $r(X)$ is contained in coker $C$ we have $r(X)=\operatorname{coker} C$. Assume now that coker $A$ is of infinite dimension and that $r(X)$ is not dense in coker $C$. We are going to construct a nest $N$ on $H$ with the property $I=I_{-}$and such that $A A^{*}$ is in $F_{r}(N)$ and $C C^{*}$ is not in $F_{r}(N)$. Let $\left\{e_{n}\right\}_{n=1}^{\infty}$ be an orthonormal basis of $\operatorname{coker} A$. We set $P_{n}=\left[e_{m}\right.$ : $m \leq n]$ for $n=1,2, \ldots$. We set $Q_{n}=r(A)^{\perp}+A P_{n}$. Let $N$ be the nest $\left\{Q_{n}\right.$ : $n=1,2, \ldots\} \cup\{H,\{0\}\}$. It is clear that $N$ satisfies $I=I_{-}$. Now $A_{\infty}^{-1}\left(Q_{n}\right)=$ $\operatorname{Ker} A+P_{n}$ for $n=1,2, \ldots$, and so $A^{-1}(\operatorname{Pr} N)$ being equal to $\operatorname{Ker} A+\bigcup_{n=1}^{\infty} P_{n}$, is dense in $H$. It follows from Theorem 5 that $A A^{*}$ is in $F_{r}(N)$. Put $R_{n}=X\left(P_{n}\right)$ for $n=1,2, \ldots$. We have $Q_{n}=r(A)^{\perp}+C R_{n}$, which by condition i) is equal to $r(C)^{\perp}+C R_{n}$, and so $C^{-1}\left(Q_{n}\right)=\operatorname{Ker} C+R_{n}$ for $n=1,2, \ldots$. It follows that $C^{-1}(\operatorname{Pr} N)=\operatorname{Ker} C+\bigcup_{n=1}^{\infty} R_{n}$. But then $C^{-1}(\operatorname{Pr} N)$ is contained in $\operatorname{Ker} C+r(X)$. Since $r(X)$ is not dense in $\operatorname{coker} C$, we see that $C^{-1}(\operatorname{Pr} N)$ is not dense in $H$. Hence, by Theorem $5, C C^{*}$ is not in $F_{r}(N)$.

Note that condition b)i) does not imply condition a). One can see that in the example constructed by Douglas in [9, p.120]. Also it is easy to see that condition b)ii) does not imply condition a). In fact, let $A$ be an operator in $B(H)$ such that $r(A)$ is not dense in $H$. Put $X=A$, and $C=I$. Then we have $A=C X$, $\operatorname{coker} X=\operatorname{coker} A$ and $r(X)$ is contained in coker $C$. Clearly b)ii) is satisfied. But since $r(A)$ is not dense in $H$, condition a) is not satisfied.

Theorem 23 is not valid for general nests. This is shown in the following example.

Example 1. Let $H_{1}$ be a Hilbert space and $N_{1}$ be a nest in $H_{1}$ such that $I=$ $I_{-}$. Take a unit vector $\xi$ in $H$ which is not $N$-proper. Put $A=\xi \otimes \xi$. Then $A^{-1}\left(\operatorname{Pr} N_{1}\right)=[\xi]^{\perp}$, and hence $A^{-1}\left(\operatorname{Pr} N_{1}\right)$ has codimension one in $H_{1}$.

Let $H_{2}$ be a Hilbert space and $\left\{e_{n}\right\}_{n=1}^{\infty}$ an orthonormal basis of $H_{2}$. Set $\eta=$ $\sum_{n=1}^{\infty} n^{-1} e_{n}$ and define the operator $B$ by $B=\sum_{n=1}^{\infty} n^{-1} e_{n} \otimes e_{n}$. Then $r(B)$ is dense in $H_{2}$. Take an orthonormal basis $\left\{f_{n}\right\}_{n=1}^{\infty}$ of $[\eta]^{\perp}$. Put $P_{m}=\left[f_{1}, f_{2}, \ldots, f_{m}\right]$ for 
$m=1,2, \ldots$, and consider the nest

$$
N_{2}=\left\{[\eta]+\left(P_{m}\right)^{\perp}: m=1,2, \ldots\right\} \cup\{[\eta],\{0\}, H\} .
$$

Note that $B^{-1}([\eta])=\{0\}$.

Put $H=H_{1} \oplus H_{2}$ and define a nest $N$ on $H$ by $N=N_{1} \cup\left\{H_{1} \oplus P: P \in N_{2}\right\}$. Note that $\bigcup_{P \in N, P=P_{-}} P=H_{1}$.

Define operators $X$ and $Y$ in $B(H)$ by $X x=A x$ if $x$ is in $H_{1}, X x=B x$ if $x$ is in $H_{2}, Y x=A x$ if $x$ is in $H_{1}, Y x=x$ if $x$ is in $H_{2}$. Then $X=Y X$, and $r(X)$ is dense in coker $Y$. Now put $R=H_{1}+[\eta]$. Then $n_{R}(X)=1$ and $n(X)=1$. It now follows from Theorem 5 that $X X^{*}$ is in $F_{r}(N)$. On the other hand, for every $S$ in $N, S>H_{1}$, we have $n_{S}(Y)=0$ and $n(Y)=1$. It follows from Theorem 5 that $Y Y^{*}$ is not in $F_{r}(N)$. Hence Theorem 23 does not hold for general nests.

Theorem 23 has some useful corollaries.

Corollary 24. Let $N$ be a nest on $H$ such that $I=I_{-}$. Let $A$ and $C$ be positive operators in $B(H)$. Put $A_{1}=A^{\frac{1}{2}}, C_{1}=C^{\frac{1}{2}}$. Assume that:

i) There exists a positive number $\lambda$ such that $A \leq \lambda C$.

ii) $\operatorname{Ker} A=\operatorname{Ker} C$.

iii) There exists a positive number $\mu$ such that $A \geq \mu C_{1} A C_{1}$.

Then if $A$ is in $F_{r}(N), C$ is in $F_{r}(N)$.

Proof. It follows from [10, Th.1] that there exists an operator $X$ in $B(H)$ such that $A_{1}=C_{1} X$, coker $X=\operatorname{coker} A_{1}$ and $r(X)$ contained in $\operatorname{coker} C_{1}$. It follows from Theorem 23 that in order to prove the assertion it suffices to show that $r(X)$ is dense in coker $C_{1}$. Assume the contrary. Since $k e r X^{*}=r(X)^{\perp}$, there exists a non-zero vector $y$ in $\operatorname{ker} X^{*} \cap \operatorname{coker} C_{1}$. Since coker $C_{1}=\overline{r\left(C_{1}\right)}$, there exists a sequence $\left\{y_{n}: n=1,2, \ldots\right\}$ such that $C_{1} y_{n}$ converges to $y$. Then the sequence $\left\{A_{1} y_{n}: n=1,2, \ldots\right\}$ converges to 0 , since $A_{1} y_{n}=X^{*} C_{1} y_{n}$ and $y$ is in $\operatorname{ker} X^{*}$. We have $\left\langle A y_{n}, y_{n}\right\rangle \geq\left\langle\mu C_{1} A C_{1} y_{n}, y_{n}\right\rangle$, and hence

$$
\left\langle A_{1} y_{n}, A_{1} y_{n}\right\rangle \geq \mu\left\langle A_{1} C_{1} y_{n}, A_{1} C_{1} y_{n}\right\rangle
$$

for $n=1,2, \ldots$. Taking limits we find that $0 \geq \mu\left\langle A_{1} y, A_{1} y\right\rangle$, which implies that $A_{1} y=0$. This is a contradiction, since $y$ belongs to $\operatorname{coker} C_{1}=\operatorname{coker} A_{1}$. Hence $r(X)$ is dense in $\operatorname{coker} C_{1}$.

Corollary 25. Let $N$ be a nest on $H$ such that $I=I_{-}$. Let $A$ and $C$ be positive operators in $B(H)$. Assume that:

a) There exists a positive number $\lambda$ such that $A \leq \lambda C$.

b) $\operatorname{Ker} A=\operatorname{KerC}$.

c) $A$ and $C$ commute.

Then if $A$ is in $F_{r}(N), C$ is in $F_{r}(N)$.

Proof. Put $\mu=\|C\|^{-1}$. The conclusion follows from Corollary 24 .

The following corollary answers a question posed by Shields in [23].

Corollary 26. Let $N$ be a nest on $H$ such that $I=I_{-}$. Let $A$ be a positive operator in $B(H)$ and $0<\lambda \leq 1$. Then if $A$ is in $F_{r}(N), A^{\lambda}$ is in $F_{r}(N)$.

Proof. Without loss of generality we may assume that $A$ is a contraction. The conclusion then follows from Corollary 25. 
Corollary 26 does not hold if we assume $\lambda \geq 1$. This is shown in the following example.

Example 2. We are going to show that there exist a nest $N$ such that $I=I_{-}$and a positive operator $B$ in $B(H)$ with the following properties:

a) $B$ is in $F_{r}(N)$.

b) $B^{2}$ is not in $F_{r}(N)$.

Let $H$ be a Hilbert space and $\left\{e_{n}\right\}_{n=0}^{\infty}$ an orthonormal basis of $H$. We set $P_{n}=\left[e_{m}: m \leq n\right]$ for $n=0,1,2, \ldots$ Let $N$ be the nest $\left\{P_{n}: n=0,1,2, \ldots\right\} \cup$ $\{H,\{0\}\}$. Set $\xi=\sum_{n=1}^{\infty} n^{-1} e_{n}$ and $A=\sum_{n=1}^{\infty} n^{-1} e_{n} \otimes e_{n}+\xi \otimes e_{0}$. Put $B=A A^{*}$. Then $B=\sum_{n=1}^{\infty} n^{-2} e_{n} \otimes e_{n}+\psi \otimes e_{0}+e_{0} \otimes \psi+c e_{0} \otimes e_{0}$, where $\psi=\sum_{n=1}^{\infty} n^{-2} e_{n}$ and $c$ is a positive number. The set $A^{-1}(\operatorname{Pr} N)$ is dense in $H$, because it contains $e_{n}$ for $n=0,1,2, \ldots$. It follows from Theorem 5 that $B$ is in $F_{r}(N)$. Let $x=\sum_{n=0}^{\infty} x_{n} e_{n}$ be in $B^{-1}(\operatorname{Pr} N)$. Then $B x$ is $N$-proper. The coefficient of $e_{n}$ in $B x$ is $n^{-2}\left(x_{n}+x_{0}\right)$. Since $B x$ is $N$-proper, there exists $n_{0}$ such that $x_{n}+x_{0}=0$ for $n \geq n_{0}$. This implies that $x_{0}=0$, and so $B^{-1}(\operatorname{Pr} N)$ is orthogonal to $e_{0}$. It follows from Theorem 5 that $B^{2}$ is not in $F_{r}(N)$.

Proposition 27. Let $N$ be a nest on $H$ such that $I=I_{-}$. Let $A$ and $C$ be operators in $F_{r}(N)$. Then $A+C$ is in $F_{r}(N)$.

Proof. Put $A_{1}=A^{\frac{1}{2}}, C_{1}=C^{\frac{1}{2}}$. It follows from Theorem 2.2. of [11] that $r\left(A_{1}\right)+$ $r\left(C_{1}\right)=r\left((A+C)^{\frac{1}{2}}\right)$. By Theorem $13 r\left(A_{1}\right)$ and $r\left(C_{1}\right)$ are in $O R(N)$, and so $r\left(A_{1}\right)+r\left(C_{1}\right)$ is in $O R(N)$ by Theorem 22 . Using Theorem 13 again, we see that $A+C$ is in $F_{r}(N)$.

The following proposition characterises the positive operators with closed range which belong to $F_{r}(N)$. We say that a subspace $V$ of $H$ is $N$-proper if $\operatorname{V} \cap \operatorname{Pr} N$ is dense in $V$.

Proposition 28. Let $N$ be a nest such that $I=I_{-}$. Let $A$ be a positive operator in $B(H)$ with closed range. Then $A$ is in $F_{r}(N)$ if and only if $r(A)$ is $N$-proper.

Proof. Put $A_{1}=A^{\frac{1}{2}}$. It is easy to see that since $r(A)$ is closed, $r(A)=r\left(A_{1}\right)$ and hence $r\left(A_{1}\right)$ is closed. By [2, Prop. 6], $\left(A_{1}\right)^{-1}(\operatorname{Pr} N)$ is dense in $H$ if and only if $r\left(A_{1}\right)$ is $N$-proper. The proposition now follows from Theorem 5 .

The following theorem provides a decomposition of a positive operator $A$ into a "factorable" and a "completely non-factorable" part with respect to a nest $N$ satisfying $I=I_{-}$. An analogous decomposition has been obtained in [21] and [1] for special cases.

Theorem 29. Let $N$ be a nest such that $I=I_{-}$. Let $A$ be a positive operator in $B(H)$. There exist operators $B$ and $C$ in $B(H)$ with the following properties:

a) $B \geq 0, C \geq 0, A=B+C$.

b) $B$ is in $F_{r}(N)$.

c) If $E$ is in $A l g N$ and satisfies $E E^{*} \leq C$, then $E=0$.

d) If $F$ is in Alg $N$ and satisfies $F F^{*} \leq A$, then $F F^{*} \leq B$.

Moreover, the operators $B$ and $C$ are unique with these properties. 
Proof. Put $A_{1}=A^{\frac{1}{2}}$. Put $H_{1}=\overline{\left(A_{1}\right)^{-1}(\operatorname{Pr} N)}$ and let $R$ be the orthogonal projection onto $H_{1}$. Define $B_{1}=A_{1} R, C_{1}=A_{1} R^{\perp}$. Then $A_{1}=B_{1}+C_{1}$ and $B_{1}\left(C_{1}\right)^{*}=0$. We show that $r\left(C_{1}\right) \cap \operatorname{Pr} N=\{0\}$. Let $x$ be a vector in $H$ such that $C_{1} x$ is in $\operatorname{Pr} N$. Then $A_{1} R^{\perp} x$ is in $\operatorname{Pr} N$, and so $R^{\perp} x$ is in $H_{1}$ and hence it is 0 . Since $C_{1} x$ $=C_{1} R^{\perp} x$, we conclude that $C_{1} x=0$.

Put $B=B_{1}\left(B_{1}\right)^{*}, C=C_{1}\left(C_{1}\right)^{*}$. We have $B \geq 0, C \geq 0$ and $A=A_{1}\left(A_{1}\right)^{*}=$ $B_{1}\left(B_{1}\right)^{*}+C_{1}\left(C_{1}\right)^{*}=B+C$. So a) is satisfied. We show that b) is satisfied. The space $\left(A_{1}\right)^{-1}(\operatorname{Pr} N)+R^{\perp} H$ is dense in $H$ and is contained in $\left(B_{1}\right)^{-1}(\operatorname{Pr} N)$. Therefore $\left(B_{1}\right)^{-1}(\operatorname{Pr} N)$ is dense in $H$, and it follows from Theorem 5 that $B$ is in $F_{r}(N)$.

Assume $E$ is in $A l g N$ and satisfies $E E^{*} \leq C$. Then $r(E) \subseteq r\left(C_{1}\right)$ by [10]. But $r\left(C_{1}\right) \cap \operatorname{Pr} N=\{0\}$. Since $E^{-1}(\operatorname{Pr} N)$ is dense in $H, E=0$.

Now we prove that d) is satisfied. By [10] there exists a contraction $X$ in $B(H)$ such that $F=A_{1} X$. The operator $X$ sends $F^{-1}(\operatorname{Pr} N)$ into $\left(A_{1}\right)^{-1}(\operatorname{Pr} N)$, and since $F^{-1}(\operatorname{Pr} N)$ is dense in $H$ we conclude that the range of $X$ is contained in $H_{1}$. Therefore $F=A_{1} X=\left(A_{1} R+A_{1} R^{\perp}\right) X=A_{1} R X$. So $F F^{*} \leq B$.

Assume now that the operators $B_{0}$ and $C_{0}$ have the properties a) through d). Since $B=D D^{*}$ for some $D$ in $A \lg N$, it follows from d) that $B \leq B_{0}$. Similarly $B_{0} \leq B$, and so $B=B_{0}$ and $C=C_{0}$.

Using the results of this section, one may obtain analogous results for the set $F_{l}(N)$ for a nest $N$ with the property $0=0_{-}$.

\section{AKNOWLEDGEMENT}

The authors wish to thank A. Katavolos for useful discussions during the preparation of this paper.

\section{REFERENCES}

[1] Adams, G. T., Froelich, J., McGuire, P. J. and Paulsen, V. I., Analytic reproducing kernels and factorization, Indiana U. Math. J. 43 (1994), 839-856. MR 95k:47027

[2] Anoussis, M. and Katsoulis, E. G., Factorisation in nest algebras, Proc. Amer. Math. Soc. 125 (1997), 87-92. MR 97c:47053

[3] Arveson, W. B., Analyticity in operator algebras, Amer. J. Math. 89 (1967), 578-642. MR 36:6946

[4] Arveson, W. B., Interpolation problems in nest algebras, J. Funct. Anal. 53 (1983), 208-233. MR 52:3979

[5] Conway, J. B., A course in functional analysis, Springer-Verlag (1985). MR 86h:46001

[6] Davidson, K. R., Nest algebras, Pitman Research Notes in Mathematics Series, 191 (1988). MR 90f: 47062

[7] Devinatz, A., The factorization of operator valued functions, Ann. Math. 73 (1961) 458-495. MR 23:A3997

[8] Dixmier, J., Étude sur les varietés et les opérateurs de Julia, Bull. Soc. Math. France 77 (1949), 11-101. MR 11:369f

[9] Douglas, R. G., On factoring positive operator functions, J. Math. Mech. 16 (1966) 119-126. MR 35:782

[10] Douglas, R. G., On majorization, factorization and range inclusion of operators in Hilbert space, Proc. Amer. Math. Soc. 17 (1966), 413-416. MR 34:3315

[11] Fillmore, P. A. and Williams, J. P., On operator ranges, Advances in Math. 7 (1971), 254-281. MR 45:2518

[12] Foias, C., Invariant para-closed subspaces, Indiana U. Math. J. 21 (1972), 887-906. MR 45:2516 
[13] Gohberg, I. C. and Krein, M. G., Theory and applications of Voltera operators in Hilbert space, Transl. Math. Monographs, 24 (1970), AMS. MR 41:9041

[14] Kadison, R. V. and Ringrose J. R., Fundamentals of the theory of operator algebras, Vol. I, Academic Press, (1983). MR 85j:46099

[15] Larson, D. R., Nest algebras and similarity transformations, Ann. Math. 121 (1985), 409-427. MR 86j:47061

[16] Lowdenslager, D. B., On factoring matrix valued functions, Ann. Math. 78 (1963), 450-454. MR 27:5094

[17] McAsey, M., Muhly, P. and Saito, K-S., Nonselfadjoint crossed products (invariant subspaces and maximality), Trans. Amer. Math. Soc. 248 (1979) 381-410. MR 80j:46101b

[18] Muhly, P., The function-algebraic ramifications of Wiener's work on prediction theory and random analysis, in: Norbert Wiener: Collected Works with Commentaries, Vol. III, The MIT Press, Cambridge, Mass., (1981) 339-370. MR 83i:01089

[19] Pitts, D. R., Factorization problems for nests: Factorization methods and caracterizations of the universal factorization property, J. Funct. Anal. 79 (1988), 57-90. MR 90a:46160

[20] Power, S. C., Factorization in analytic operator algebras, J. Funct. Anal. 67 (1986), 413-432. MR 87k: 47040

[21] Power, S. C., Spectral characterization of the Wold-Zasuhin decomposition and predictionerror operator, Math. Proc. Camb. Phil. Soc. 110 (1991), 559-567. MR 92j:47032

[22] Ringrose, J. R., On some algebras of operators, Proc. London Math. Soc. (3) 15 (1965), 61-83. MR 30:1405

[23] Shields, A. L, An analogue of a Hardy-Littlewood-Fejer inequality for upper triangular trace class operators, Math. Z. 182 (1983), 473-484. MR 85c:47022

Department of Mathematics, University of the Aegean, Karlovassi, Samos 83200 Greece

E-mail address: mano@aegean.gr

Department of Mathematics, East Carolina University, Greenville, North Carolina 27858

E-mail address: makatsov@ecuvm.cis.ecu.edu 